\title{
NONADDITIVITY OF MASKING BY NARROW-BAND NOISES
}

\author{
Mark E. Perkins and Michael S. Landy \\ Department of Psychology and Center for Neural Science, New York University, New York, \\ NY 10003, U.S.A.
}

(Received 21 August 1989; in revised form 4 April 1990)

\begin{abstract}
Characterization of the visual system as a linear system has many consequences. One property implied by such a characterization is that signal-to-noise ratio at threshold is constant, so that the contrast energy of a sinusoidal grating at threshold depends on the effective noise passed by the filter used to detect the target. When the contrast energy in an external noise is sufficiently high, the contribution of internal noise may be conveniently ignored. In such circumstances, the effectiveness of a given noise is measured by its ability to mask the target. One consequence of the linearity assumption is that if the energy of the effective noise is increased by a factor $k$, then threshold energy of the signal is increased by the same amount. The contrast energy at threshold in the presence of a masker created by adding two maskers should be the sum of the threshold energies in the individual maskers. We have tested this hypothesis for spectrally nonoverlapping maskers. We find that the contrast energy required to detect the target in the presence of the combined maskers is much greater than the sum of the threshold energies for the two maskers. This "excess masking" violates the linearity assumption. On the other hand, when maskers do have spectral overlap, no excess masking is found.
\end{abstract}

Masking Linearity Spatial pattern detection

\section{INTRODUCTION}

An attractive hypothesis is that the visual system initially analyzes the visual input using a bank of linear bandpass spatial filters (e.g. Wilson \& Bergen, 1979; Wilson \& Giese, 1977). However, in recent years, evidence has accumulated which suggests that substantial nonlinearities are involved at a relatively early stage. This evidence is both psychophysical (Chubb \& Sperling, 1988; Legge \& Foley, 1980; Wilson, 1980) and physiological (e.g. at the simple cell level: Dean, Tolhurst \& Walker, 1982; Reid \& Shapley, 1988; Tolhurst, Walker, Thompson \& Dean, 1980). Even a simple nonlinear transducer function following linear oriented channels may be insufficient to account for spatial pattern masking results (Derrington \& Henning, 1989). On the other hand, Jamar and Koenderink (1985) have found that it is possible to use effective spectral (contrast) energy over a broad range of spatial frequency to predict performance at detection of noise gratings, consistent with a linear channels hypothesis.

The experiments we report in this paper suggest that, under certain conditions, masking of a sinusoidal grating by narrow-band noise behaves in a nonlinear fashion. One consequence of the linearity assumption is that the contrast energy required to detect a signal in a noisy background depends on the total noise energy passed by the filter being used to detect the target, assuming that the noise level is sufficiently high so that the contribution of internal noise is negligible (Henning, Hertz \& Hinton, 1981; Pelli, 1981; Stromeyer \& Julesz, 1972). Specifically, the ratio of signal energy at threshold to total noise energy passed by the filter (i.e. effective noise energy) is constant. For example, if one simply doubles the noise energy, then threshold should also double, as has been amply demonstrated (Pelli, 1981; Stromeyer \& Julesz, 1972). This notion is illustrated schematically in Fig. 1.

In Fig. 1A we represent a target sinusoid with frequency $f$ and a filter centered at $f$. Threshold contrast is represented by the height of the arrow. It is commonly assumed that an observer uses a filter centered on the target frequency under these conditions. Of course, it is also possible that the subject uses a filter which is not centered on the target frequency (off-frequency looking, see expt 2), or pools across multiple channels (see the Discussion section). Figure 1B 

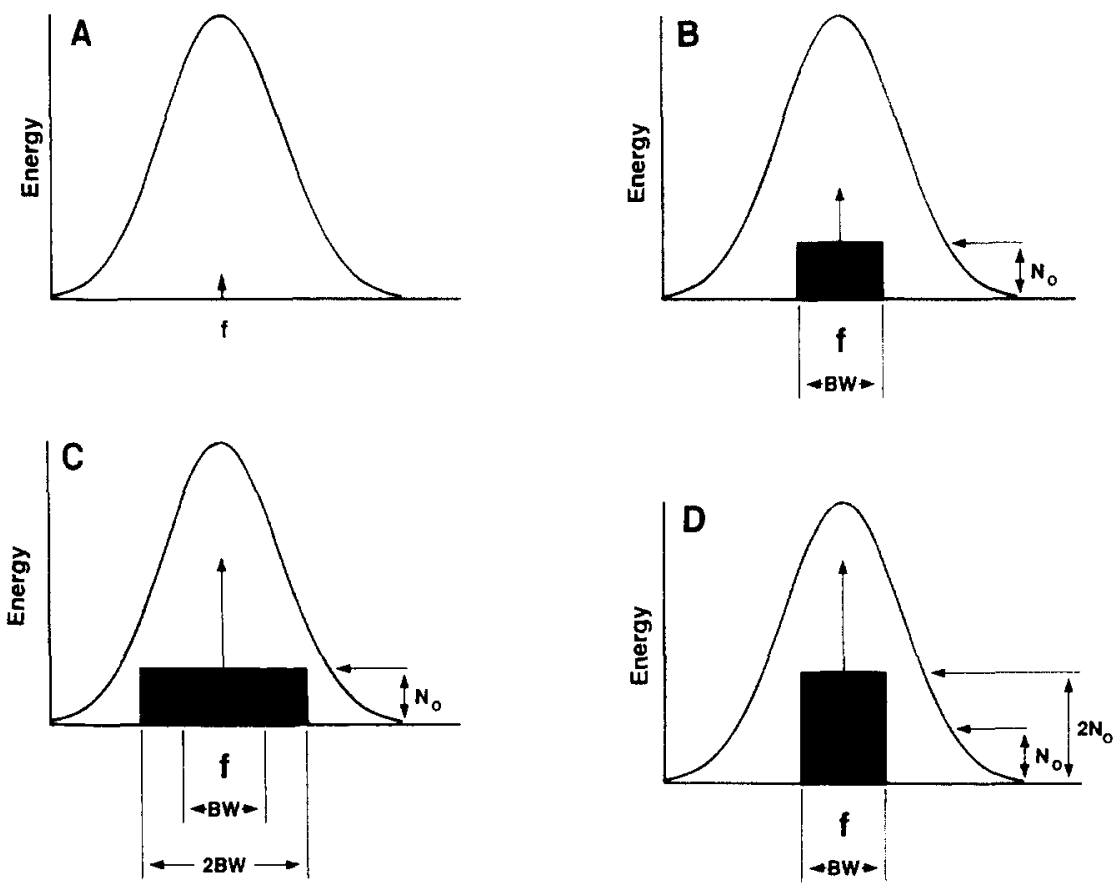

Fig. 1. Schematic representation of masking. In all panels, the subject is required to detect a target sinusoid of frequency $f$. The length of the arrow at $f$ indicates the threshold contrast required for detection. (A) Simple detection. The curve indicates a filter used by the subject to perform the detection task. (B) An experiment in which the observer is to detect this target in a background of bandpass noise with bandwidth $B W$ and noise spectral density $N_{0}$. The total noise energy is given by the product of bandwidth and noise spectral density. (C) Noise spectral density remains constant and effective bandwidth (that passed by the filter) is doubled. (D) Bandwidth is held constant and noise spectral density is doubled. In both (C) and (D) signal energy at threshold, represented by the height of the arrow, is doubled (constant signal-to-noise ratio).

represents a situation in which the observer is to detect this target in a background of bandpass noise. The noise has bandwidth $B W$ and noise spectral density $N_{0}$. The total noise energy is given by the product of bandwidth and noise spectral density. The fact that the rectangle representing the noise is completely under the filter curve is intended to illustrate that the entire noise band is passed by the filter. In other words, the effective noise is the entire noise band. There are a number of ways to manipulate experimental conditions in order to increase the threshold energy for the target (e.g. by a factor of 2). Two possibilities are shown in Fig. $1 C$ and D. In Fig. 1C, noise spectral density remains constant and bandwidth is doubled. In Fig. 1D, this situation is reversed. Of course, for plausible filters, doubling the bandwidth would be insufficient to double threshold given the gradual falloff of the filter sensitivity at frequencies remote from the center frequency. In any case, the important point is that threshold for the target will be doubled as the amount of noise passed by the filter is doubled, regardless of the manner in which noise passed by the filter is increased. Pelli (1981) and Stromeyer and Julesz (1972) performed experiments which confirmed linearity with increases in noise power for a single noise band (Fig. 1D), along with bandwidening experiments of the sort illustrated in Fig. 1C (to measure the shape of the filter used by the subjects).

In audition it has been observed that, under fairly general experimental conditions, masking of a target by a compound masker, created by combining two or more simple maskers, is greater than the sum of the individual masking effects (e.g. Green, 1967; Lutfi, 1983). This effect requires that the two simple maskers have no spectral overlap (Lutfi, 1985). In other words, there must be a spectral gap between the two simple maskers in order to see this excess masking effect. We set out to investigate the extent to which similar effects exist in vision when sinusoidal gratings are masked by narrow-band noises.

Figure 2 represents such a compound masking paradigm. This is the design of our first 

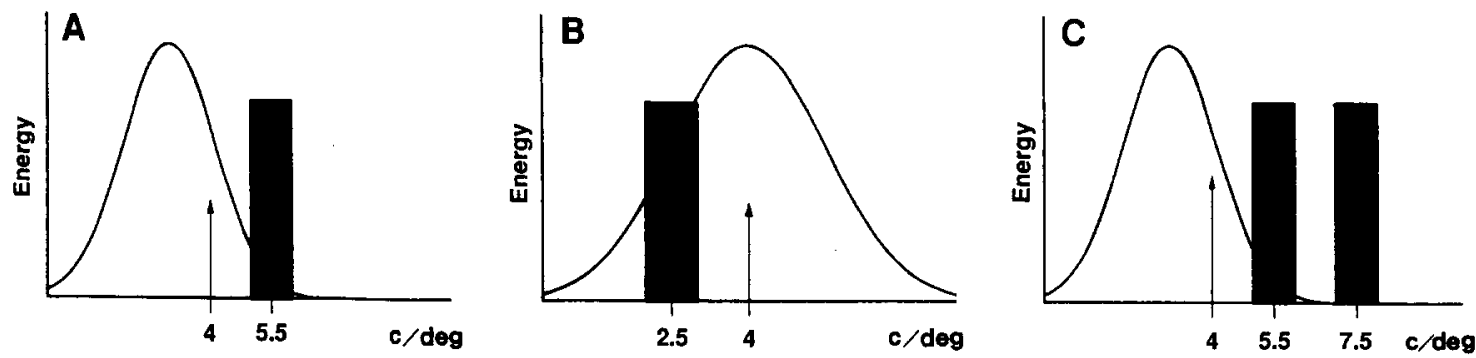

Fig. 2. Representation of masking by noises with energy that does not overlap the target frequency (expt 1). (A) A $4 \mathrm{c} / \mathrm{deg}$ target in the presence of a narrow-band noise, $1 \mathrm{c} / \mathrm{deg}$ wide, centered at $5.5 \mathrm{c} / \mathrm{deg}$. Target energy at threshold is indicated by the height of the arrow. (B) A noise band centered at $2.5 \mathrm{c} / \mathrm{deg}$. For simplicity, target threshold energy is represented as being the same as that in (A). (C) The configuration when these two noises are combined. If the system is linear and the observer uses the same filter to detect the target in all of these maskers, then threshold energy for the target should be twice that in either masker alone.

experiment. Figure $2 \mathrm{~A}$ illustrates the presentation of $4 \mathrm{c} / \mathrm{deg}$ target in the presence of a narrow-band noise, $1 \mathrm{c} / \mathrm{deg}$ wide, centered at $5.5 \mathrm{c} / \mathrm{deg}$. Target energy at threshold is again indicated by the height of the arrow. Figure 2B depicts a similar situation in which the noise band is centered on $2.5 \mathrm{c} / \mathrm{deg}$. By a suitable choice of noise level and center-frequency, threshold for the target can be made to be the same as in Fig. 2A. If we combine these two noises as shown in Fig. $2 \mathrm{C}$, and the system is linear, and the observer uses the same filter in all situations, then threshold energy for the target should be twice that in either masker alone. It is important to note that there is nothing special about doubling the noise energy passed through this filter. If the two thresholds are different, the linear theory predicts that threshold energy in the combined masker will be given by the sum of the individual threshold energies.

\section{METHODS}

Methods were the same for all experiments. Only the spectral content of the noises changed. Noises and targets were generated using the HIPS image-processing software (Landy, Cohen \& Sperling, 1984a, b). They were computed in advance of the experiment and stored on disk.

\section{Noises}

All noises were dynamic, with 60 uncorrelated noise frames per sec. Noises were one-dimensional (constant in the vertical dimension). They were filtered with digital approximations to 10th-order Butterworth bandpass filters, resulting in a bandpass characteristic with steep spectral decline at both ends of the band.

\section{Target}

The target was a vertical sinusoidal grating with a spatial frequency of $4 \mathrm{c} / \mathrm{deg}$.

\section{Stimulus presentation}

Stimuli were presented on a monochrome monitor (U.S. Pixel PX15) with a fast P4-like phosphor, viewed from a distance of $75 \mathrm{~cm}$. Stimuli subtended $4.2 \times 4.2 \mathrm{deg}$. Mean luminance was $40 \mathrm{~cd} / \mathrm{m}^{2}$. Target and noise were windowed identically in space and in time using Gaussian envelopes. Spatial tapering was in the horizontal direction only (the stimuli were one-dimensional and did not vary in the vertical direction). The maximum target contrast was 0.13 and the maximum peak-to-peak noise contrast was 0.35 . The horizontal space constant, defined as the distance between the $1 / e$ points on the Gaussian envelope, was $1.4 \mathrm{deg}$. The time constant, similarly defined, was $167 \mathrm{msec}$.

Presentation of stimuli, timing of experimental events, and collection of responses were controlled by an Adage RDS-3000 display system. The refresh rate was $60 \mathrm{~Hz}$, noninterlaced. We used a modification of the procedure described by Watson, Nielsen, Poirson, Fitzhugh, Bilson, Nguyen and Ahumada (1986) for precise contrast control. Target and noise were interleaved in space and in time. On even-numbered frames of a stimulus sequence, noise was displayed on the even-numbered lines of the CRT. On odd-numbered frames of a stimulus sequence, noise was displayed on the odd-numbered lines. When the presentation was noise alone, the other lines were at mean luminance (i.e. $40 \mathrm{~cd} / \mathrm{m}^{2}$ ). When signal-plus-noise was desired, the nonnoise lines on the display had the 
windowed sinusoidal grating. The spacing of successive raster lines of the display was $2 \mathrm{~min}$. Thus, the noise and signal were each vertically subsampled at 30 samples per deg, where these samples jittered from frame to frame (first odd lines, then even lines) at $30 \mathrm{~Hz}$. This high-frequency subsampling and jitter were not visible to the subjects.

The subject viewed the CRT display through a circular aperture, which was slightly larger than the square stimuli, in a matte gray screen. At the beginning of a session, the subject adjusted the illumination on this screen so that it matched the mean luminance of the display, which was constant throughout the experiment. For each block of trials, the type of noise was fixed (including noise band and energy level). Thresholds were measured using a two-alternative forced-choice (2AFC) adaptive procedure (Levitt, 1970). Target contrast increased after one incorrect response and decreased after two consecutive correct responses, tracking a performance level of $70.7 \%$ correct. Signal contrast changed in steps of approx. 0.003. The last 10 turn-around points in a block of trials were averaged to give an estimate of threshold. At least three blocks of trials were run at each combination of noise band and noise energy. The first block of each condition was always discarded as practice. In some cases (especially early in the experiment), there was a clear learning effect observed in the estimated thresholds. Pelli (1981) also found learning effects in experiments in which off-frequency looking was a useful strategy (as we discuss below). In such cases, more than three blocks were run. In all cases, the last two blocks of each condition were used to estimate thresholds reported here.

\section{Timing of a trial}

Each trial was preceded by a cue spot of $250 \mathrm{msec}$ duration, followed by a $167 \mathrm{msec}$ blank interval. Each stimulus interval had a duration of $500 \mathrm{msec}$, as measured between the 3 SD points of the Gaussian temporal envelope. Stimulus intervals were separated by an interstimulus interval of $333 \mathrm{msec}$. Subjects responded by pressing a button indicating which interval they thought contained the signal. After the subject responded, auditory feedback indicated which interval contained the signal. The next trial was initiated automatically by the subject's response.

\section{EXPERIMENT 1}

Contrast threshold for the $4 \mathrm{c} / \mathrm{deg}$ sinusoidal target was measured at several noise levels for each of three masker configurations: (1) a narrow-band noise ( $1 \mathrm{c} / \mathrm{deg}$ at half-height) centered at $2.5 \mathrm{c} / \mathrm{deg}$; (2) a narrow-band noise (1 c/deg at half-height) centered at $5.5 \mathrm{c} / \mathrm{deg}$; (3) a masker created by combining these noises. The linear model of contrast detection requires the contrast energy at threshold for the target in the combined masker to be the sum of the threshold contrast energies for the individual maskers, assuming that the same filter is used by the subject for all three conditions. If the individual thresholds are identical, then energy at threshold in the combined masker should be twice that in the individual maskers.

The results of this experiment for the two subjects are shown in Fig. 3. The abscissa is the noise spectral density of a single noise band, on a logarithmic scale. In the case of the singleband maskers, this corresponds to the total energy of the noise, since the bandwidth was $1 \mathrm{c} / \mathrm{deg}$. The total energy in the combined masker is twice the number shown on the abscissa. The left ordinate is the (Michelson) contrast of the target at threshold, also on a log scale, and the right ordinate is a logarithmic scale of the contrast energy of the target at threshold. The two sets of open symbols, which are virtually superimposed for subject MEP, represent thresholds in the presence of the single-band maskers. Clearly, these maskers are equally effective in masking the signal for subject MEP, and nearly so for subject SMW. The solid lines through these points correspond to constant signal-to-noise ratio. For subject MEP, a single line has been fitted to both sets of points. For subject SMW, individual lines have been fitted to each set of points. The dashed line in each figure is the prediction for the combined masker conditions based on additivity of masking. For subject MEP, it is a factor of 2 increase (along the energy axis at the right of the figure) from the solid line fitted to the individual maskers. For subject SMW, the dashed line is the energy sum of the two solid lines fitted to the data. The solid symbols are thresholds in the combined masker. The line through these points corresponds to constant signal-to-noise ratio. For subject MEP, this line is a factor of 10.8 increase from the individual maskers. In other words, there is excess masking 


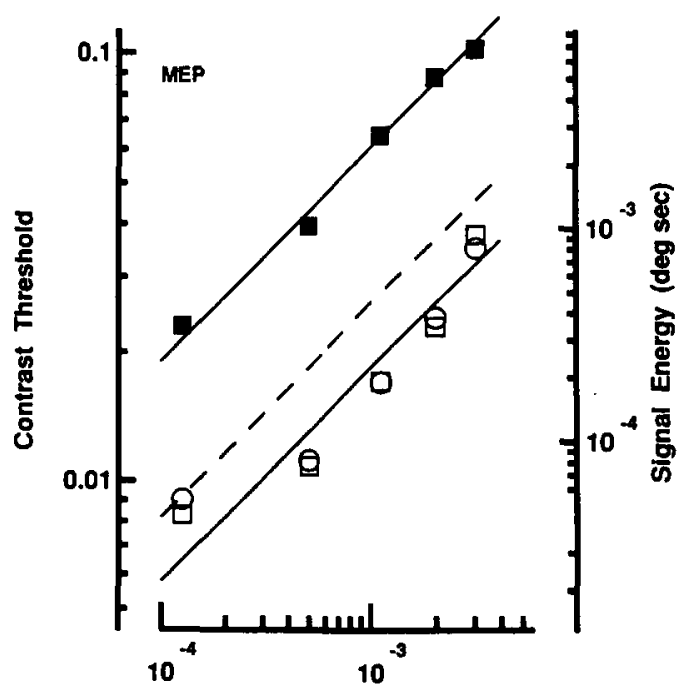

Noise Spectral Density (deg sec)

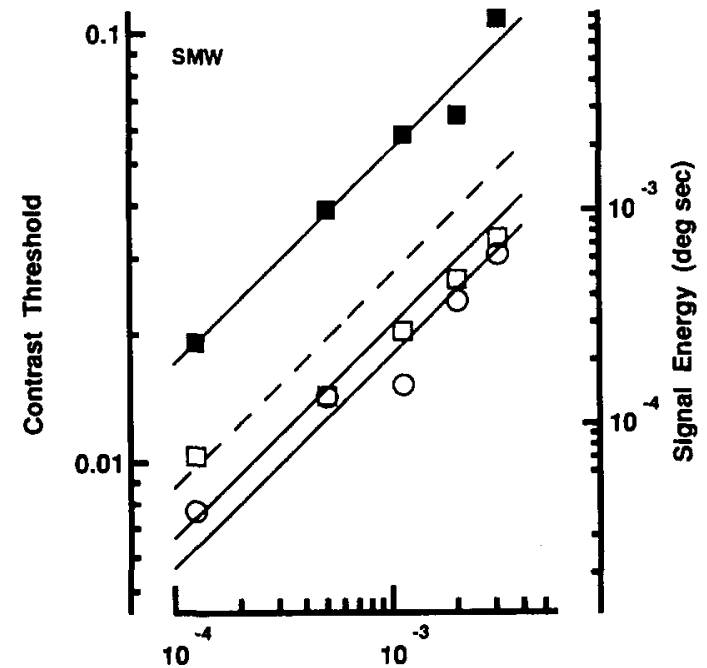

Noise Spectral Density (deg sec)

Fig. 3. Results of expt 1 for two subjects. The abscissa is the noise spectral density of a single noise band, on a logarithmic scale. This corresponds to the total energy of the noise for the single-band maskers. The total energy in the combined masker is twice the number shown on the abscissa. The left ordinate is the (Michelson) contrast of the target at threshold, also on a log scale, and the right ordinate is a logarithmic scale of the contrast energy of the target at threshold. $(O, \square)$ Thresholds in the presence of the single-band maskers $(O=$ noise centered at $2.5 \mathrm{c} / \mathrm{deg}, \square=$ noise centered at $5.5 \mathrm{c} / \mathrm{deg})$. The solid lines through these points correspond to constant signal-to-noise ratio. For the first subject, a single line was fit to both sets of single-band noise masking data; for the second subject separate lines were fit to each single-band condition. The dashed line is the prediction of additivity of masking. It is a factor of two increase (along the energy axis at the right of the figure) from the single solid line fitted to both individual masker conditions for the first subject, and the sum of the individual fits for the second. ( $\boldsymbol{G}$ ) Thresholds in the combined masker. The line through these points corresponds to constant signal-to-noise ratio. For the first subject, it is a factor of 10.8 increase from the individual maskers, giving excess masking by a factor of 5.4 when the target is masked by spectrally flanking maskers. For the second subject the excess masking is by a factor of 3.8. Standard errors are generally on the order of the plotted symbol size or smaller in this and later figures. Note that the open circles and open squares overlap extensively for the middle three noise levels for subject MEP, and for the second noise level for subject SMW.

by a factor of 5.4 when the target is masked by spectrally flanking maskers. For subject SMW, the excess masking is by a factor of 3.8 . Both subjects show excess masking. These effects are not as large as those seen in the auditory literature (Lutfi, 1983), but are nevertheless quite substantial.

\section{EXPERIMENT 2}

One possible explanation of the results of expt 1 is that it is possible for the observer to adopt a strategy of off-frequency looking, in which the observer uses a filter centered at a frequency other than the target frequency in order to improve the signal-to-noise ratio as passed by that filter. With narrow-band noise, the observer may improve performance by using a filter centered spectrally on the other side of the signal from the masking noise. An example is shown in Fig. $4 \mathrm{~A}$ where the target is at $4 \mathrm{c} / \mathrm{deg}$ and the masker is centered at $5.5 \mathrm{c} / \mathrm{deg}$. An observer might improve the signal-to-noise ratio by using a filter centered somewhat below $4 \mathrm{c} / \mathrm{deg}$. For the $2.5 \mathrm{c} / \mathrm{deg}$ masker, the subject might use a filter centered above $4 \mathrm{c} / \mathrm{deg}$ to improve signal-to-noise ratio. When the masker is composed of flanking noise bands, as shown in Fig. 4B, off-frequency looking no longer provides improved signal-to-noise ratio. Thus, the use of off-frequency looking only when such a strategy is useful (the single masker conditions) could explain the excess masking found in expt 1. Numerous studies in psychoacoustics (e.g. Leshowitz \& Wightman, 1971; O'Loughlin \& Moore, 1981) have demonstrated that such off-frequency observation is a viable strategy for performing many detection tasks. Pelli (1981) has collected data investigating off-frequency looking in the presence of high- and low-pass noises, but we know of no investigations for narrow-band noises. 

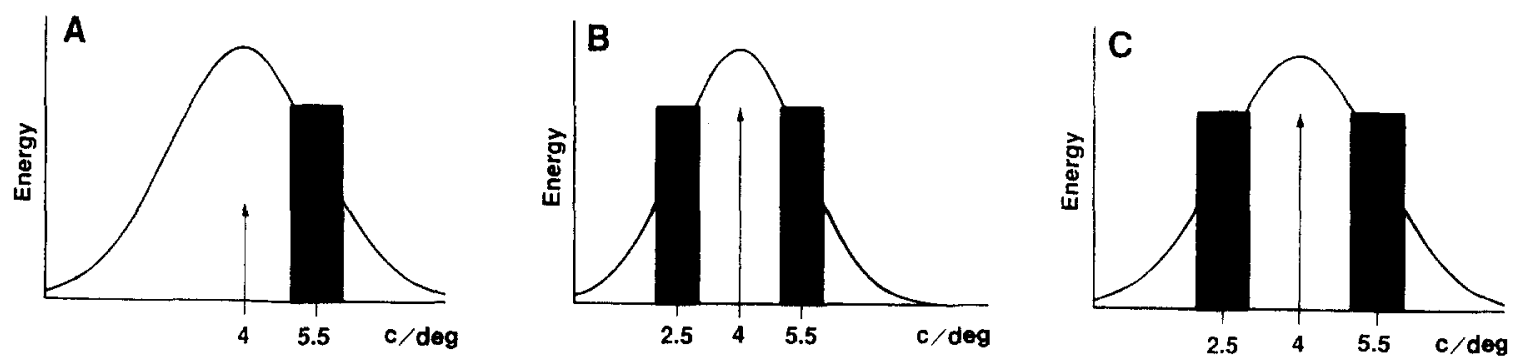

Fig. 4. Representation of off-frequency looking as a strategy for detection. (A) A target at $4 \mathrm{c} / \mathrm{deg}$ and masker centered at $5.5 \mathrm{c} / \mathrm{deg}$. The observer might improve signal-to-noise ratio by using a filter centered somwhere below $4 \mathrm{c} / \mathrm{deg}$. (B) The masker is composed of flanking noise bands; off-frequency looking no longer provides improved signal-to-noise ratio. (C) The masker configuration used in expt 2 . Two noise bands, separated by a spectral gap, were both at higher spatial frequencies than the signal. In such a situation, any advantage afforded by off-frequency looking is still available with the combined masker.

To test whether the excess masking can be attributed to off-frequency looking, we used the masker configuration shown in Fig. 4C in which two noise bands, separated by a spectral gap, were both at higher spatial frequencies than the signal. Specifically, the masker centered at $5.5 \mathrm{c} / \mathrm{deg}$ was paired with a masker centered at $7.5 \mathrm{c} / \mathrm{deg}$, and the maskers were separated by a spectral gap. In such a situation, any advantage afforded by off-frequency looking for the individual maskers is also available for the combined masker. However, if the excess masking is indeed due to unusual processing of maskers with spectral gaps, as suggested in the auditory literature, then this masker configuration should still reveal it.
With the combined masker, since all of the masker energy is on the high-frequency side of the signal, it should still be possible to make use of a filter centered on a frequency lower than the signal. If off-frequency looking plays the dominant role, then either additivity will be observed or there may be no change in threshold when the masker at $7.5 \mathrm{c} / \mathrm{deg}$ is included (subadditivity).

We performed such an experiment using maskers at frequencies higher than the target spatial frequency. Contrast threshold was measured at the same noise levels as in expt 1 . The three masker configurations were: (1) a narrow-band noise $(1 \mathrm{c} / \mathrm{deg}$ at half-height) centered at $5.5 \mathrm{c} / \mathrm{deg}$; (2) a narrow-band noise
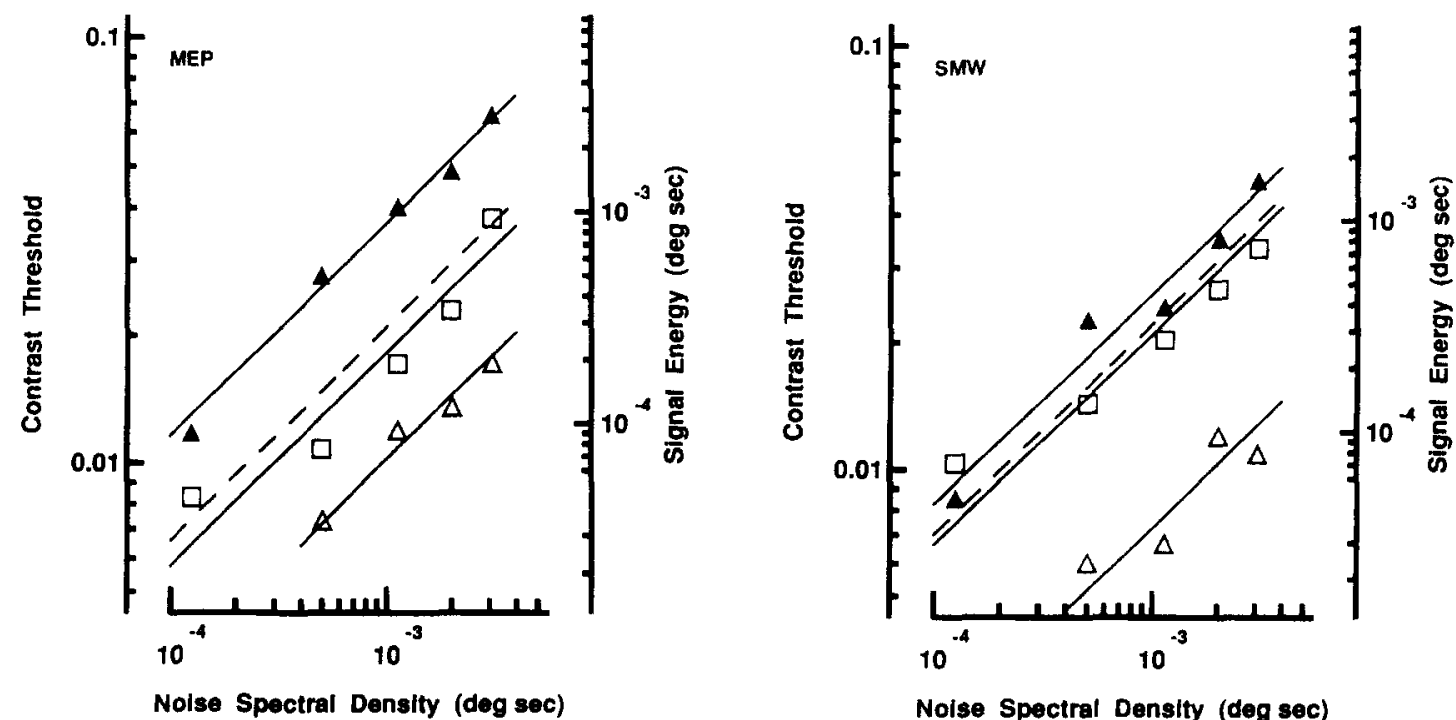

Fig. 5. Results for expt 2 for two subjects. Abscissa and ordinate are as in Fig. 3. ( $\triangle$ ) Thresholds in the masker centered at $7.5 \mathrm{c} / \mathrm{deg}$. ( $\square$ ) Thresholds replotted in the masker at $5.5 \mathrm{c} / \mathrm{deg}$ from expt 1 . The solid lines through these data correspond to constant signal-to-noise ratio. The dashed line is the prediction of the additive model of masking. (A) Thresholds obtained in the combined masker, the line drawn through them also corresponds to constant signal-to-noise ratio. The vertical distance between this line and the dashed line represents the excess masking for this masker configuration. For the two subjects, the excess masking is by a factor of 3 and 1.4 , respectively. 
( $1 \mathrm{c} / \mathrm{deg}$ at half-height) centered at $7.5 \mathrm{c} / \mathrm{deg}$; and (3) a masker created by combining these noises. Results are shown in Fig. 5. Open triangles mark thresholds in the masker centered at $7.5 \mathrm{c} / \mathrm{deg}$. Open squares replot thresholds in the masker at $5.5 \mathrm{c} / \mathrm{deg}$ from expt 1 . Again, the solid lines correspond to constant signal-tonoise ratio. The dashed line is the prediction of the additive model of masking. Thresholds obtained in the combined masker are marked by solid triangles, and the line drawn through them also corresponds to constant signal-to-noise ratio. In this case, subject MEP shows excess masking by a factor of three above the prediction based on additivity. Subject SMW shows excess masking by a factor of approx. 1.4. There are large subject differences in the relative effects of the two individual maskers and in the amount of excess masking, but both subjects do show significant excess masking (recall that the standard errors in the figures are generally smaller than the data points). It appears, then, that the superadditive effect of maskers separated by spectral gaps is not due solely to off-frequency looking, although it could explain a portion of the excess masking.

\section{EXPERIMENT 3}

We performed one further experiment to assess the extent to which the excess masking

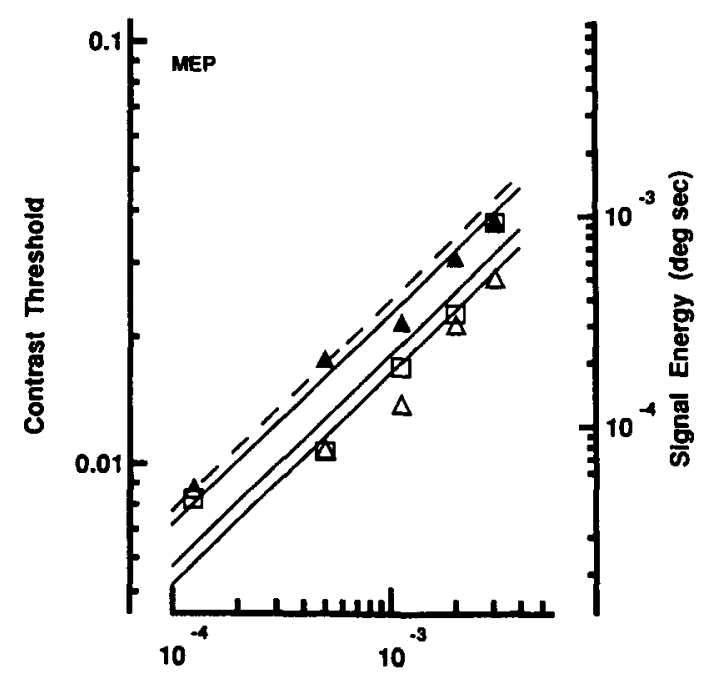

Nolse Spectral Density (deg sec) can be attributed to the fact that the individual maskers do not overlap (the spectral gap present in the combined maskers in the previous two experiments). In the auditory literature, this excess masking effect only occurs with compound maskers involving a spectral gap between masker components (Lutfi, 1985). We measured contrast threshold in the presence of a masker centered at $6.5 \mathrm{c} / \mathrm{deg}$ with a $1 \mathrm{c} / \mathrm{deg}$ bandwidth. We also measured threshold in $2 \mathrm{c} / \mathrm{deg}$ bandwidth noise centered on $6 \mathrm{c} / \mathrm{deg}$. This noise can be thought of as the sum of two $1 \mathrm{c} / \mathrm{deg}$ bandwidth noises, one centered on $5.5 \mathrm{c} / \mathrm{deg}$ and the other centered on $6.5 \mathrm{c} / \mathrm{deg}$. This is identical to increasing the bandwidth of a narrow-band noise. If the excess masking can be attributed to the existence of a spectral gap in the noise, then additivity of masking should obtain.

The results of this experiment are shown in Fig. 6. The open symbols represent thresholds in the individual maskers, and the solid symbols represent thresholds in the wide-band masker created by combining the narrow-band maskers. Solid lines correspond to constant signal-tonoise ratio, and the dashed line is the prediction of the additive model of masking. For both subjects, thresholds in the wide-band masker are slightly less than that predicted by the additive model. For subject SMW, the compound masker data consistently fall below the data for the $5.5 \mathrm{c} / \mathrm{deg}$ masker data. This is unexpected

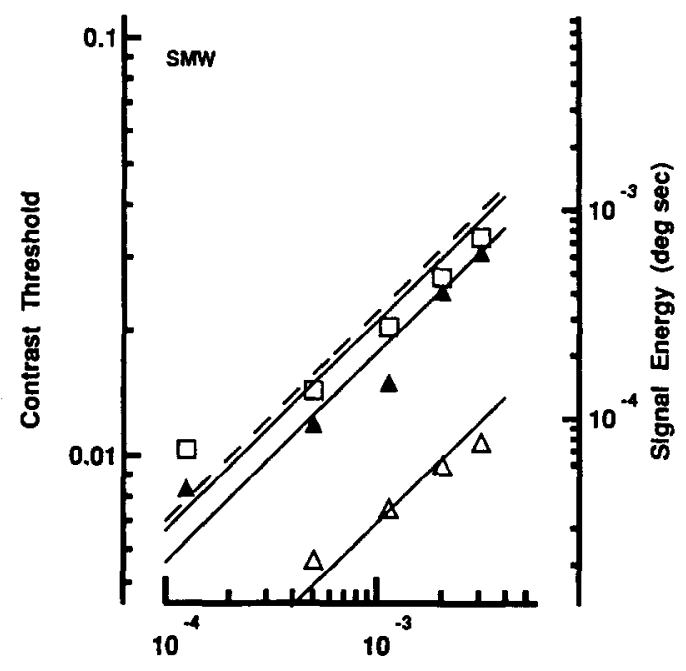

Noise Spectral Density (deg sec)

Fig. 6. Results of expt 3 for two subjects. Abscissa and ordinate are as in Fig. 3. $(\triangle$ ) Thresholds in the masker centered at $6.5 \mathrm{c} / \mathrm{deg}$. ( $\square$ ) Thresholds replotted in the masker at $5.5 \mathrm{c} / \mathrm{deg}$ from expt 1 . (A) Thresholds in the wider-band masker $(2 \mathrm{c} / \mathrm{deg}$ bandwidth centered at $6 \mathrm{c} / \mathrm{deg})$. Solid lines correspond to constant signal-to-noise ratio. The dashed line is the prediction of the additive model of masking. Thresholds in the wider-band masker are slightly less than predicted by the additive model. For subject MEP, there are several places where plot symbols overlap and may be hard to distinguish: lowest noise level (all three conditions), second noise level ( $\square$ and $\Delta$ ), and highest noise level $(\square$ and $\boldsymbol{\Delta}$ ). 
and may be attributable to practice effects since the compound masker data were collected several months after the $5.5 \mathrm{c} / \mathrm{deg}$ masker trials. Again, there were large differences between the relative effects of the two single maskers for the two subjects. But, for both subjects there appears to be no excess masking, and some indication of subadditivity (although the possible practice effects must be taken into account).

In fact, some subadditivity is to be expected. The wide-band noise was generated by filtering broad-band noise with a $2 \mathrm{c} / \mathrm{deg}$ wide filter centered at $6 \mathrm{c} / \mathrm{deg}$, rather than a sum of the noises centered at 5.5 and $6.5 \mathrm{c} / \mathrm{deg}$. The individual noise maskers were not filtered using ideal bandpass filters, but rather had smoothly sloped skirts. The sum of the individual maskers would have had a slight bump at $6 \mathrm{c} / \mathrm{deg}$ which was not present in the masker used in the experiment. The end result is that the wide-band masker has slightly less contrast energy than the sum of the narrower maskers. Finally, we note a surprising difference between the two subjects. For subject SMW, the $6.5 \mathrm{c} / \mathrm{deg}$ masker was less effective than the $5.5 \mathrm{c} / \mathrm{deg}$ masker in increasing the threshold for the $4 \mathrm{c} / \mathrm{deg}$ target. For MEP, the two maskers had nearly identical effects. It is not clear why the more distant masker did not have a weakened effectiveness for this subject.

\section{DISCUSSION}

We measured threshold in the presence of various (single) bands of noise. In most conditions, it was found that signal-to-noise ratio at threshold is constant (this point is discussed further below). We then measured thresholds in various combinations of these maskers. Again, signal-to-noise ratio at threshold was constant. However, additivity of masking was violated. If additivity were to hold, threshold in a combination of maskers would be the sum of the thresholds in the individual maskers. Rather, we observed an increase in masking beyond that predicted by the additivity assumption. This observation holds for maskers in which a spectral gap is found, and the effect is diminished if the two maskers are both greater in spatial frequency than the target. On the other hand, when masker energy is increased by increasing the bandwidth of the masker, there was no indication of excess masking (although some of the subadditivity found here may have been due to practice effects, as already noted).
How might one explain the excess masking we have found? For expt 1, the excess masking effect merely may have been the result of an off-frequency looking strategy being used in the single masker conditions, but unavailable as a strategy for the compound masker conditions. However, expt 2 indicated that off-frequency looking was not the entire explanation. A linear model, possibly combined with varying subject strategies, does not appear to explain the failures of additivity we have found. Thus, we next examine the simplest possible nonlinear model, shown in Fig. 7. First, the contrast function of the stimulus is computed,

$$
\frac{L(x, y)-L_{\text {mean }}}{L_{\text {mean }}}
$$

followed by a nonlinear stage. Then, a series of linear filters are applied to the output of the nonlinearity. The energy at the output of these filters is computed, followed by a decision maker, which generates a response. For simplicity, we will illustrate a square-law as the nonlinear stage. The properties of such a system are well known (see, e.g. Davenport \& Root, 1957) and provide a convenient illustration of the effects of a contrast nonlinearity. As we shall see, the addition of a contrast nonlinearity to

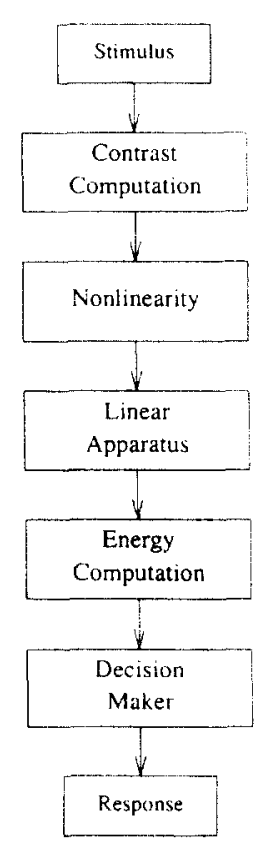

Fig. 7. Qualitative model for the results of the experiments. The contrast function of the stimulus is computed, followed by a nonlinearity. Following the nonlinearity is a collection of parallel filters with fixed bandwidth. The energy at the output of these filters is computed. A decision maker uses the energy at the output of a single filter to generate a response. 
A

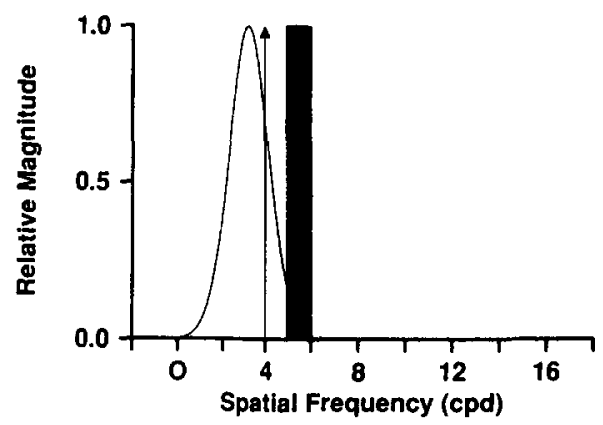

C

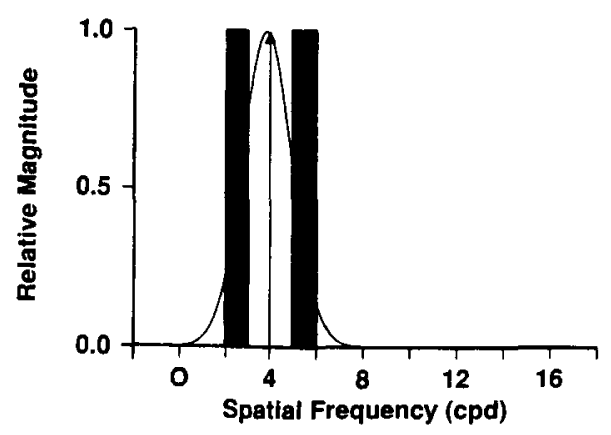

E

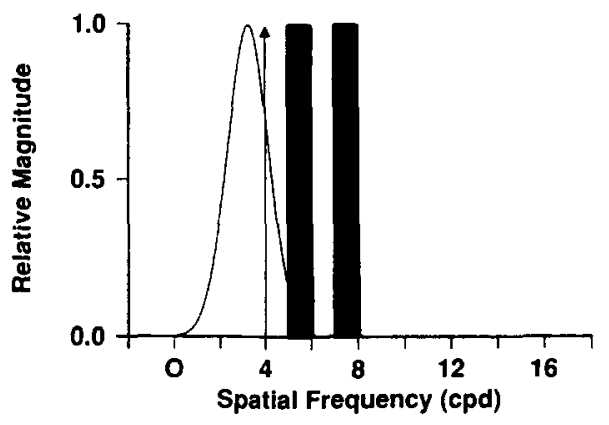

G

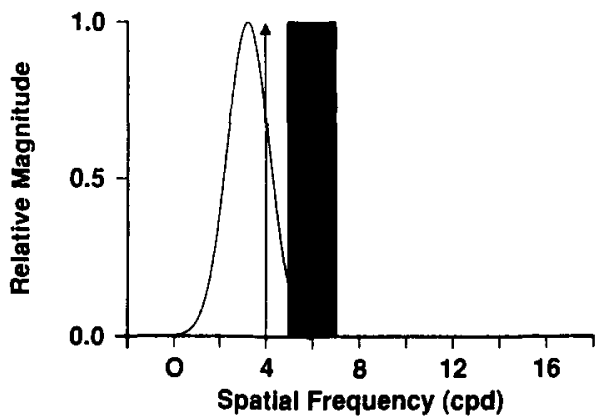

B

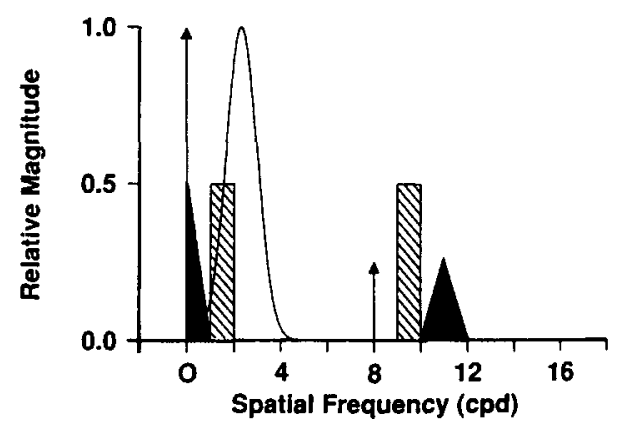

D

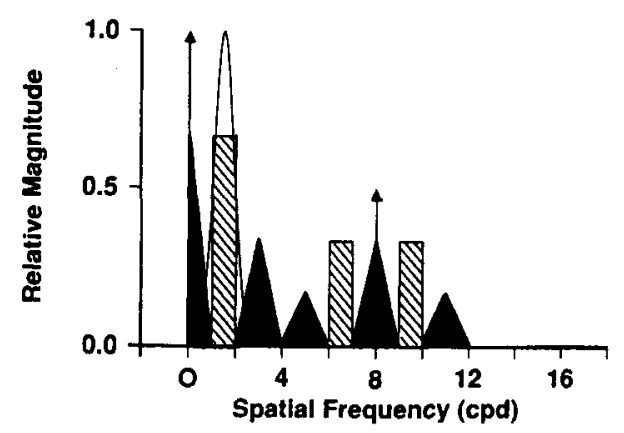

F

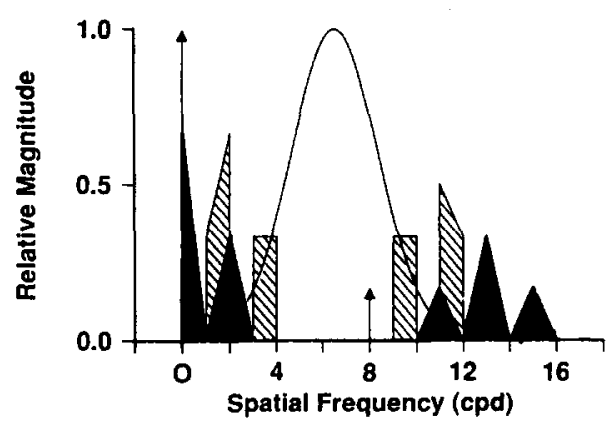

H

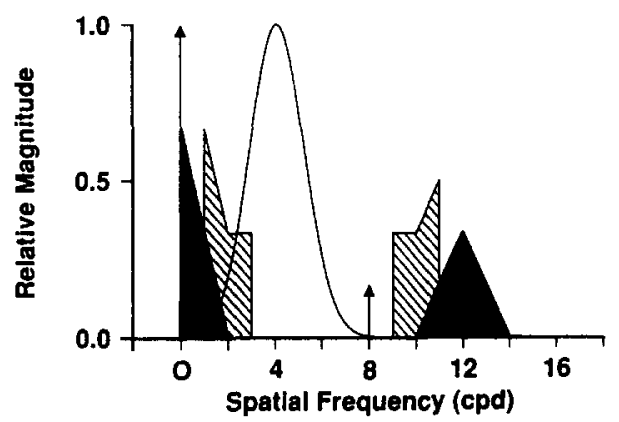

Fig. 8. The effect of squaring the contrast function. (A) The magnitude spectrum of an ideal signal at $4 \mathrm{c} / \mathrm{deg}$ and an ideal noise centered at $5.5 \mathrm{c} / \mathrm{deg}$. (B) The magnitude spectrum of the squared signal-plusnoise shown in (A). Heavy lines and darker areas indicate the parts of the spectrum which are attributable to the noise alone. The lighter lines and areas are the spectral components due to the signal-plus-noise. In both panels the optimal filter available to the subject is shown (see text for details). (C) and (D) give a similar representation for the case of spectrally flanking maskers; $(E)$ and $(F)$ depict the combined masker from expt 2; $(G)$ and $(H)$ represent the band-widening used in expt 3. 
our original linear hypothesis is not consistent with the results of our experiments, and a complete explanation of our results remains to be discovered.

Figure 8 illustrates the effect of computing the square of the contrast function of the stimulus:

$$
\left(\frac{L(x, y)-L_{\text {mean }}}{L_{\text {mean }}}\right)^{2}
$$

Figure $8 \mathrm{~A}$ shows the magnitude spectrum of an ideal signal at $4 \mathrm{c} / \mathrm{deg}$ and an ideal noise band centered at $5.5 \mathrm{c} / \mathrm{deg}$. Also shown is a hypothetical filter for use in performing the detection task using an off-frequency looking strategy. Figure $8 \mathrm{~B}$ shows the spectrum of the square of the signal-plus-noise. The heavy lines and darkly shaded areas show the parts of the spectrum which are due to the noise alone. The lighter lines and lightly shaded areas show the effect of adding the $4 \mathrm{c} / \mathrm{deg}$ signal. In this case, it is the two impulses and the two rectangular bands. With the squared stimulus, the subject need no longer look for the veridical $4 \mathrm{c} / \mathrm{deg}$ target. In fact, it is no longer present in the spectrum of the squared stimulus. Instead, the subject may use any manifestation of the target in this spectrum. This includes the harmonic distortion of the $4 \mathrm{c} / \mathrm{deg}$ target alone (at d.c. or at $8 \mathrm{c} / \mathrm{deg}$ ), or the distortion products (often called "beats") of the target interacting with the noise mask. The hypothetical filter drawn here depicts a strategy in which the subject attends to the noise "beat" band from 1 to $2 \mathrm{c} / \mathrm{deg}$, with a modicum of off-frequency looking in order to lessen the effects of the noise from d.c. to $1 \mathrm{c} / \mathrm{deg}$.

In Fig. 8C and D, we show a similar representation of the case where the signal is flanked by bands of noise. In $8 \mathrm{D}$, the subject can choose a filter centered on the noise "beat" band from 1 to $2 \mathrm{c} / \mathrm{deg}$. Figure $8 \mathrm{E}$ and $\mathrm{F}$ represent the case where both maskers are higher in frequency than the signal. In this case, a subject might look for the noise "beats" at 3.5 and at $7.5 \mathrm{c} / \mathrm{deg}$. Figure $8 \mathrm{G}$ and $\mathrm{H}$ illustrate the band-widening case of expt 3.

We have tested this class of models using the ideal noise bands illustrated in Fig. 8. We generated the expected energy spectra of noise and signal-plus-noise for the linear case (Fig. 8A, C, $\mathrm{E}$ and $\mathrm{G}$ ), and the square-law case (Fig. 8B, D, $F$ and $H$ ), along with various additive mixtures of these. Next, we computed the expected output energy from 1- and 2-octave wide filters (Gaussian in linear spatial frequency) posi- tioned at all points along the spatial frequency axis. Note that we are assuming a constant logarithmic bandwidth for the filters available to the subject. This allowed us to calculate the signal-to-noise ratio at threshold for a variety of models in each of our masking conditions. In the simulations, a constant small amount of "internal noise" was added to the outputs of all filters. Without this internal noise, and with the maskers on one side of the signal and linear processing (Fig. 8A, E and G), the model could achieve arbitrarily high signal-to-noise ratio by using a filter which was excessively far from the signal (signal passed by the filter is very low for such a filter placement, but signal-to-noise ratio is high). In the subsequent discussion we will abbreviate the three experiments as $2.5+5.5$ (the flanking maskers of expt 1), $5.5+7.5$ (the nonoverlapping maskers of expt 2), and $5.5+6.5$ (the band-widening used in expt 3 ).

The case of a linear model (omit the nonlinearity in Fig. 7) is illustrated in Fig. 9. If a filter centered on $4 \mathrm{c} / \mathrm{deg}$ is used, indeed if any single fixed filter is used, then the effects of masking are additive, as we stated earlier. This may be seen in Fig. 9, where the curve for the $2.5+5.5$ condition is the sum of the curves for the individual masker conditions (labeled 2.5 and 5.5). If instead the optimal off-frequency looking strategy is used for each condition (the

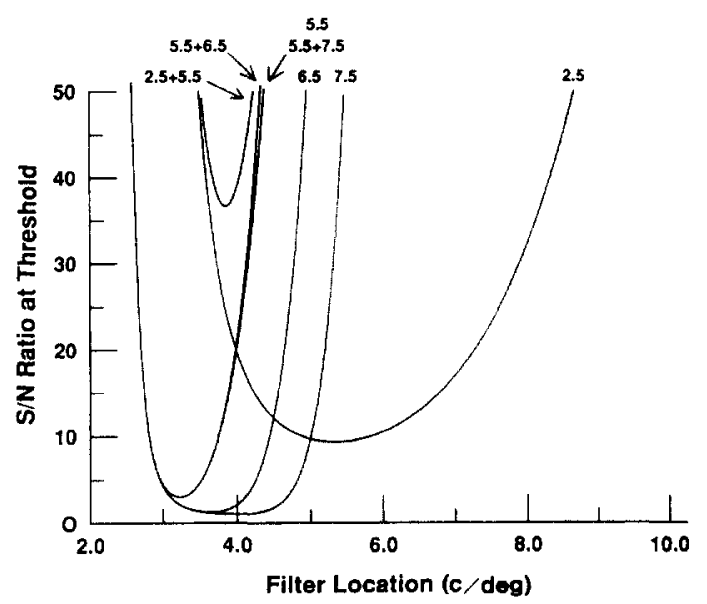

Fig. 9. Effects of filter placement. Signal to noise ratio is plotted for each simulated experiment as a function of the center frequency of a one octave wide filter used to perform the task using the linear stimulus spectra (Fig. 8A, C, E and G). An arbitrary scale factor was applied uniformly to the signal-to-noise ratios. Each curve corresponds to a different noise masker condition. Additivity for any fixed choice of filter may be demonstrated by noting that the curves labeled 2.5 and 5.5 sum to the curve labeled $2.5+5.5$. Optimal off-frequency looking implies using the lowest signal-tonoise ratio available in each curve. 
minimum of each curve in Fig. 9), then masking is no longer additive. We determined the optimal strategy by simulating the use of filters centered at all positions from 0.01 to $16 \mathrm{c} / \mathrm{deg}$ (at $0.01 \mathrm{c} / \mathrm{deg}$ steps), and chose the filter yielding the highest signal-to-noise ratio. Figure 8 indicates the optimal filter placement in each panel. With optimal filter placement and a linear model, masking is superadditive for the case of flanking maskers $(2.5+5.5)$. However, subadditivity is found for both other conditions $(5.5+7.5$ and $5.5+6.5)$.

For the case of a square-law nonlinearity, there is no longer any signal at $4 \mathrm{c} / \mathrm{deg}$, and the spectral energy at $8 \mathrm{c} / \mathrm{deg}$ which results from the signal alone is far weaker than the beats between the signal and the noise. Therefore, we only discuss the optimal off-frequency looking strategy. The optimal strategy in Fig. 8B and D is to position the filter near the noise beat from 1 to $2 \mathrm{c} / \mathrm{deg}$, but in Fig. $8 \mathrm{~F}$ it is preferable to attend to the higher noise beat as well (by placing the filter between the two, somewhat favoring the higher-frequency beat from 9 to $10 \mathrm{c} / \mathrm{deg}$ ). The lower frequency beat is favored in Fig. $8 \mathrm{H}$. Signal-to-noise calculations predict superadditivity for $2.5+5.5$, but also predict more severe superadditivity for $5.5+6.5$, and even more for $5.5+7.5$. This is not what was found experimentally.

The model fares no better for additive mixtures of the linear square-law spectra (corresponding to nonlinearities of the form $\left.a x+b x^{2}\right)$. But, there is a further problem with this line of thought. One result of our experiments is that signal energy at threshold is proportional to masker energy-signal-to-noise ratio at threshold is constant-for all of our compound masker conditions (although the data for the single masker conditions are not always that well fit by a 45 deg line; see below). This result is predicted by the linear model, since the variance of the distribution of energy passed by the filter is doubled when noise is doubled, so that a doubling of signal strength is required to overcome it. This is also predicted by the square-law model, where doubling noise quadruples the filter output, and doubling the signal quadruples the extra filter output, resulting in the same $d^{\prime}$. However, this property will no longer hold for a general polynomial nonlinearity.

Hence, the class of models represented by Fig. 7 do not easily capture the data. This is in agreement with the results of Badcock and
Derrington (1989), where motion of beats could not be explained as merely a nonlinear distortion product. It is not clear to us what alternative models would work better. If no constraints were made on the filter shape used by the subject, then the optimal strategy is to use a single filter tailored to the target and noise configuration to yield maximal signal-to-noise ratio. Any mixture of two filters results in sub-ideal performance. If the subject is constrained to use filters from a fixed set of "channels", it may be preferable to approximate the optimal filter by a suitable weighted sum of the given filters. Nevertheless, none of these possibilities explains the results reported here.

An alternative explanation might involve the nonlinear pooling of the output of multiple channels. If the outputs of several such nonoverlapping channels were simply squared and summed, for example, this would be equivalent to squaring the output of a single wider-band filter with spectral sensitivity which is the sum of the spectral sensitivities of the individual channels being pooled. We have also simulated a Euclidean pooling model with octave-wide channels (at 1, 2, 4, 8 and $16 \mathrm{c} / \mathrm{deg}$ ) using both the linear and squared spectra, and the results were not consistent with our data. Thus, a simple square-and-sum pooling does not appear to explain our results.

Another possible sub-optimal strategy (suggested by Jeff Lubin) is to place a single filter at the spectral center of mass of the stimulus. Since the center of mass changes with increasing signal strength, we assumed a negligible signal strength, and simulated this strategy by placing the filter at the spectral center of mass of the noise alone. With the linear spectra, this model did not predict our results at all (it was always subadditive). However, with the squared spectra, this strategy came reasonably close to qualitatively predicting our results. This model was strongly superadditive for the $2.5+5.5$ case, moderately superadditive for $5.5+7.5$, and only slightly superadditive for $5.5+6.5$. However, this is a peculiar model, since in many cases the filter is placed quite far from any spectral content due to the signal!

Some observers do report using the noise beat as a cue in some conditions (e.g. signal plus single noise band). However, we have not been able to discern from subject reports or our own observations what the difference is in the subject strategy employed in the $5.5+7.5$ case compared to the band-widening case, $5.5+6.5$. In 
our experiments, the signal was static (except for the Gaussian temporal vignette), and the noise was dynamic, allowing the subject to attend to low temporal frequencies. But, this was true in all conditions and does not easily explain our results. It may be possible to explain the results by restricting the set of center frequencies of filters available to the observer (both in spatial and temporal frequency). This latter possibility might be explored by further experiments using static signals in static noise, and dynamic signals (e.g. a sinusoid with phase changing randomly from frame to frame) in dynamic noise. This is an interesting possibility for further research.

In several of our conditions, the contrast threshold as a function of noise spectral density does not appear to be well fit by a straight line of slope one, but rather is curved. A typical graph of threshold vs noise contrast is flat at low noise contrasts (where internal noise dominates), and asymptotes to unit slope at higher noise contrasts once added noise swamps internal noise (Pelli, 1981). We see hints of this effect in several conditions (Fig. 3: subject MEP, single-band maskers at 2.5 and $5.5 \mathrm{c} / \mathrm{deg}$, subject SMW, single-band masker at $5.5 \mathrm{c} / \mathrm{deg}$; Fig. 6: subject MEP, single-band masker at $6.5 \mathrm{c} / \mathrm{deg}$ ). However, in two conditions there is also a slight indication that the slope is in fact greater than one (Fig. 3: subject MEP, single-band maskers at 2.5 and $5.5 \mathrm{c} / \mathrm{deg}$ ). One possibility is that subjects use off-frequency looking, but as noise and stimulus contrast increase, subjects can use a filter even more distant from the target frequency and still stay above the internal noise. Unfortunately, this predicts results which are concave-down as a function of masker energy, which does not fit any of our results. A full explanation of our results may require a variety of subject strategies across the different masker power and spectrum conditions. Such hypotheses may be explored by testing the extent to which the effects vanish when different contrast and/or noise band conditions are intermixed in the same block of trials.

\section{CONCLUSIONS}

We have investigated the extent to which a linear theory of masking of sinusoidal gratings by luminance noise is consistent with data obtained when a masker is constructed from simpler maskers which do not share a cutoff frequency. In such cases, we have found that the theory is inadequate. Such a theory predicts that contrast energy at threshold in a masker with a spectral gap is the sum of the energies at threshold in the individual maskers. We have found that threshold in the combined masker exceeds that predicted by the linear theory. The amount of this excess masking depends on the subject and on the masker configuration. We have measured excess masking effects between a factor of 1.4 and a factor of 5.4 .

Lutfi (1983) has observed similar excess masking effects in audition. Indeed, his experiments have served as the motivation for our work. Lutfi used various combinations of sinusoidal maskers and narrow-band noises to mask a sinusoidal target. He observed excess masking by a factor of 10-50, depending on the masker configuration. Our effects are more modest, but no less intriguing.

Acknowledgements - The work described in this paper was supported by a grant from the Office of Naval Research, Grant N00014-85-K-0077. We acknowledge the helpful comments on an earlier draft of this manuscript by Charles Chubb, Mike Hawken, John Krauskopf, Larry Maloney, Tony Movshon, and two anonymous reviewers. We would like to thank Robert Picardi for technical assistance. We would like to acknowledge several conversations with Bob Lutfi, which aided our understanding of the problems we discuss here. Portions of this work have been presented at the Annual Meeting of the Association for Research on Vision and Ophthalmology, Sarasota, Florida, 6 May, 1988, and published in abstract form (Perkins \& Landy, 1988).

\section{REFERENCES}

Badcock, D. R. \& Derrington, A. M. (1989). Detecting the displacements of spatial beats: No role for distortion product. Vision Research, 29, 731-739.

Chubb, C. \& Sperling, G. (1988). Drift-balanced random stimuli: A general basis for studying non-Fourier motion perception. Journal of the Optical Society of America A, 5, 1986-2007.

Davenport, W. B. \& Root, W. L. (1957). Introduction to the theory of random signals and noise. New York: McGraw-Hill

Dean, A. F., Tolhurst, D. J. \& Walker, N. S. (1982). Non-linear temporal summation by simple cells in cat striate cortex demonstrated by failure of superposition. Experimental Brain Research, 45, 456-458.

Derrington, A. M. \& Henning, G. B. (1989). Some observations on the masking effects of two-dimensional stimuli. Vision Research, 29, 241-246.

Green, D. M. (1967). Additivity of masking. Journal of the Acoustical Society of America, 4l, 1517-1525.

Henning, G. B., Hertz, B. G. \& Hinton, J. L. (1981). Effects of different hypothetical detection mechanisms on the shape of spatial-frequency filters inferred from masking experiments. Journal of the Optical Society of America, 71, 574-581.

Jamar, J. H. T. \& Koenderink, J. J. (1985). Contrast detection and detection of contrast modulation for noise gratings. Vision Research, 25, 511-521 
Landy, M. S., Cohen, Y. \& Sperling, G. (1984a). HIPS: A UNIX-based image processing system. Computer Vision, Graphics and Image Processing, 25, 331-347.

Landy, M. S., Cohen, Y. \& Sperling, G. (1984b). HIPS: Image processing under UNIX software and applications. Behavior Research Methods, Instruments and Computers, 16, 199-216.

Legge, G. E. \& Foley, J. M. (1980). Contrast masking in human vision. Journal of the Optical Society of America, 70, 1458-1471.

Leshowitz, B. \& Wightman, F. L. (1971). On-frequency masking with continuous sinusoids. Journal of the Acous tical Society of America, 49, 1180-1190.

Levitt, H. (1970). Transformed up-down methods in psychoacoustics. Journal of the Acoustical Society of America, 49, 467-477.

Lutfi, R. A. (1983). Additivity of simultaneous masking. Journal of the Acoustical Society of America, 73, 262-267.

Lutfi, R. A. (1985). A power-law transformation predicting masking by sounds with complex spectra. Journal of the Acoustical Society of America, 77, 2128-2136.

O'Loughlin, B. J. \& Moore, B. C. J. (1981). Off-frequency listening: Effects on psychophysical tuning curves obtained in simultaneous and forward masking. Journal of the Acoustical Society of America, 69, 1119-1125.

Pelli, D. G. (1981). Effects of visual noise. Unpublished Ph.D. dissertation, Cambridge University.
Perkins, M. E. \& Landy, M. S. (1988). Visual masking is not additive. Investigative Ophthalmology and Visual Science (Suppl.), 29, 369.

Reid, R. C. \& Shapley, R. M. (1988). Complex temporal stimuli increase relative sensitivity of cat striate cortical neurons to high temporal frequencies. Investigative Ophthalmology and Visual Science (Suppl.), 29, 23.

Stromeyer, C. F. \& Julesz, B. (1972). Spatial frequency masking in vision: Critical bands and spread of masking. Journal of the Optical Society of America, 62, 1221-1232.

Tolhurst, D. J., Walker, N. S., Thompson, I. D. \& Dean, A. F. (1980). Non-linearities of temporal summation in neurones in area 17 of the cat. Experimental Brain Research, 38, 431-435.

Watson, A. B., Nielsen, K. R. K., Poirson, A., Fitzhugh, A., Bilson, A., Nguyen, K. \& Ahumada, A. J. (1986). Use of a raster framebuffer in vision research. Behavior Research Methods, Instruments and Computers, 18, 587-594.

Wilson, H. R. (1980). A transducer function for threshold and suprathreshold human vision. Biological Cybernetics, $38,171-178$.

Wilson, H. R. \& Bergen, J. R. (1979). A four mechanism model for threshold spatial vision. Vision Research, 19, 19-32.

Wilson, H. R. \& Giese, S. C. (1977). Threshold visibility of frequency gradient patterns. Vision Research, 17, $1177-1190$. 